\title{
Microstructure of GaAs/GaN interfaces produced by direct wafer fusion
}

\author{
J. Jasinski ${ }^{1}$ and Z. Liliental-Weber \\ Materials Science Division, Lawrence Berkeley National Laboratory, Berkeley, CA 94720 \\ S. Estrada and E.Hu \\ Materials Department, University of California, Santa Barbara, CA 93106
}

Results of a Transmission Electron Microscopy (TEM) study of directly bonded GaAs/GaN structures obtained by wafer fusion are presented. A large fraction of the interface area was found to be well-bonded, with the presence of a thin (1-2 nm) amorphous layer of a native oxide at the bonded interface. The dominant defects found in non- bonded areas were cavities. Most of them were elongated along the $[110]_{\mathrm{GaAs}}$ direction and their sizes differed by three orders of magnitude in the range from a few tens of nanometers to a few tens of micrometers. Plan-view TEM study of well-bonded regions showed the presence of two dislocation networks. The first, formed at the interface to accommodate an unintentional tilt between the fused crystals, consists of a one-dimensional array of wavy dislocations located at interface steps and separated by a few tens of nanometers. The second dislocation network formed to relieve additional strain from the thermal processing of the samples, consists of a two-dimensional, irregularly-spaced grid of dislocations formed on the GaAs side, running parallel to either $[110]_{\mathrm{GaAs}}$ or $[1 \underline{10}]_{\mathrm{GaAs}}$ directions.

\footnotetext{
${ }^{1}$ E-mail: jbjasinski@1bl.gov
} 
Wafer bonding of lattice-mismatched materials has demonstrated great success in producing heterogeneous device structures that could not be formed through alternative methods, such as epitaxial growth. By integrating materials such as $\mathrm{GaAs} / \mathrm{InP}, \mathrm{InGaAs} / \mathrm{Si}$ and $\mathrm{GaAs} / \mathrm{Si}$, wafer bonding has produced a variety of devices such as light emitting diodes [1], vertical cavity lasers [2], vertical couplers [3] and avalanche photodiodes [4]. Recently, GaN wafer fusion has been used to create GaAs/GaN diodes and an n-AlGaAs/p-GaAs/n-GaN heterojunction bipolar transistor (HBT) [5], as well as to integrate GaAs- and GaN-based light-emitting heterostructures [6]. This paper reports on TEM results on the microstructure of $\mathrm{GaAs} / \mathrm{GaN}$ interfaces produced by wafer bonding.

Structures used for this study were produced by direct fusion of a GaAs wafer on top of a 2 micron thick GaN layer grown on c-plane (0001) sapphire by Metal Organic Chemical Vapor Deposition (MOCVD) (Fig.1). The GaAs layer was 1 micron thick and it was grown by Molecular Beam Epitaxy (MBE) on a $0.3 \mu \mathrm{m}$-thick AlAs etch stop layer. $10 \mu \mathrm{m}$ wide, $\sim 100 \mathrm{~nm}$ deep channels, were etched into the GaAs substrate, forming a rectangular grid of $150 \mu \mathrm{m} \times 400$ $\mu \mathrm{m}$. These were intended to facilitate the removal of gas or liquid byproducts of the fusion process. The GaAs and GaN surfaces were measured to have $<0.4 \mathrm{~nm} \mathrm{rms}$ roughness . Wafers were cleaned with acetone and isopropanol, then underwent two sequential oxidations (first by oxygen plasma then by ultra-violet-ozone) followed by oxide removal steps (in $\mathrm{NH}_{4} \mathrm{OH}$ ). Finally, GaN and GaAs were rinsed in methanol, joined together in methanol, and then fused in a nitrogen ambient under uniaxial pressure of $2 \mathrm{MPa}$. Two different bonding temperatures $\left(550^{\circ} \mathrm{C}\right.$ and $\left.750^{\circ} \mathrm{C}\right)$ and two different bonding times $(15 \mathrm{~min}$ and $60 \mathrm{~min})$ were used to produce four different samples. Further details of the growth conditions of the materials, the fusing apparatus and conditions can be found elsewhere [7]. Removal of the GaAs substrate by selective wet 
etching resulted in the following sample structure: $\operatorname{GaAs}(1 \mu \mathrm{m}) / \mathrm{GaN}(2 \mu \mathrm{m}) / \mathrm{sapphire}$. No attempt was made to optimize the twist angle between the GaN and GaAs layers and therefore each of these four samples has a different, accidental twist angle. Cross-sectional TEM specimens were prepared from the two samples fused at $750^{\circ} \mathrm{C}$ and one fused for $60 \mathrm{~min}$ at $550^{\circ} \mathrm{C}$. In the second sample fused at $550^{\circ} \mathrm{C}$ the GaAs layer separated from the GaN during specimen preparation, which suggested very weak bonding. In addition to cross- sectional specimens plan-view TEM specimens were prepared from the sample fused at $750^{\circ} \mathrm{C}$. Interface microstructure and defect distributions were studied using a Topcon 002B microscope operated at $200 \mathrm{kV}$.

All TEM studies indicated that there were no substantial differences in the microstructure of the fused interface between samples bonded at $550^{\circ} \mathrm{C}$ and $750^{\circ} \mathrm{C}$. The details of this microstructure will be described in the following.

The TEM study of cross-sectional specimens showed that most of the interface area was well bonded. A High Resolution Electron Microscopy (HREM) image of this well-bonded interface observed in the sample fused for $15 \mathrm{~min}$ at $750^{\circ} \mathrm{C}$ is shown in Fig. 2(a). Such HREM studies showed that a very thin layer of bright contrast, most likely amorphous material, was visible at the interface even in the well-bonded regions. Its thickness varied between 5-10 $\AA$ in samples bonded at $750^{\circ} \mathrm{C}$ and $15-20 \AA$ in the one bonded at $550^{\circ} \mathrm{C}$. Thin layers of residual native oxide have previously been observed in other systems of bonded materials [8,9], both by TEM and by Secondary Ion Mass Spectroscopy of the fused interface. We also confirmed the presence of oxygen at the $\mathrm{GaAs} / \mathrm{GaN}$ bonded interface by detailed energy dispersive X-ray (EDX) analysis. These details have been published elsewhere [5]. Also observed at the interface were regions with bright contrast, generally identified as 'cavities'. As in the case of GaAs/InP or $\mathrm{GaN} /$ sapphire bonded materials, these cavities were asymmetric, extended primarily into one 
material only. In the case of the $\mathrm{InP} / \mathrm{GaAs}$ the cavity always extended more deeply into the InP side $[8,10]$, while cavities formed at the fused GaAs/sapphire interface formed almost entirely in the GaAs and revealed distinct faceting on (001) and (111)-type GaAs planes [11,12]. In these $\mathrm{GaAs} / \mathrm{GaN}$ samples, the cavities extended almost entirely into the GaAs. These cavities also revealed (001) and (111)-type facets (Fig. 2(c)), although some cavities were lens-like in shape (Fig. 2(b)). The heights of the cavities ranged between 5-20 nm, whereas their horizontal dimension varied from a few tens of nanometers to a few tens of micrometers. The cavities were also evident in the plan-view samples, appearing as bright regions (small ovals on Fig. 2(d) and narrow long strips on Fig. 3) that are distributed between well-bonded areas with a characteristic line pattern. Most of the cavities were elongated along the $[110]_{\mathrm{GaAs}}$ direction. EDX measurements indicated excess oxygen within the cavities, and suggest that the cavity regions are partially empty [5]. The formation of such cavities in the GaAs/InP system was correlated with enhanced diffusion processes that took place under the bonding conditions. These cavities may be formed from the migration of small voids, resulting from surface irregularities of the wafers. During the bonding process, these voids can migrate at the interface and agglomerate to reduce the overall area of non-bonded interface. The asymmetry of the cavity shape observed in the present study can be associated with the fact that at the fusion temperature, the diffusion of vacancies takes place mainly in GaAs. Reduction of surface free energy of the system is the driving force for the observed faceting of cavity surfaces.

The plan-view samples show a series of dislocations networks that would accommodate the tilt misorientation and the thermal mismatch between GaAs and GaN. One of these networks, a one-dimensional array of irregular wavy lines, is clearly visible in Fig. 3(a). These lines are oriented at some average angle relative to the $[110]_{\mathrm{GaAs}}$ direction (the direction of the elongation 
of cavities). The angle varies from sample to sample, and also shows local variation within a sample. An average spacing between neighboring lines of this array is of the order of a few tens of nanometers. We measured average spacing of these lines of about $55 \mathrm{~nm}$ and $35 \mathrm{~nm}$ for samples fused for 1 hour and 15 min, respectively. We believe that this network accommodates the tilt between the bonded GaAs and GaN. Tilt between 2 materials brought together in wafer bonding has 2 principal components: an average, long-scale tilt originating from the miscut and local tilts due to the surface roughness and irregularities. The 'miscut tilt' dislocations will form relatively uniformly distributed steps at the interface. The direction of the dislocation lines is determined by the tilt axis, and the spacing between dislocations depends on the tilt angle. For small tilts $\omega$ it can be approximated by $D=b / \omega$ where, $b$ is a component of the Burgers normal to the bonding plane. Assuming that the Burgers vector of dislocations forming this network is of the order of $a_{\mathrm{GaAs}}$ or less we estimated that this tilt has a very small value of the order of $1^{\circ}$.

Plan-view TEM also reveals straight dislocation lines along the $[110]_{\mathrm{GaAs}}$ and $[1 \underline{10}]_{\mathrm{GaAs}}$ directions; these features are indicated between the pairs of arrows in Fig. 3. The spacing between these lines varies from place to place but they form a two-dimensional array with an average separation between lines in the range of a few micrometers. We believe that this network results from the thermal mismatch between the GaAs and GaN. Fusion takes place at an elevated temperature and the sample is subsequently cooled down to room temperature. The twodimensional network of irregularly-spaced dislocations is formed to accommodate the resulting strain. The average separation between dislocations of this network depends on the magnitude of the strain, which needs to be relaxed. Networks of such dislocations have been reported in the literature for the $\mathrm{InP} / \mathrm{GaAs}$ system $[8,13]$. They are usually observed at some distance from the interface and the average separation between dislocations measured in this system was of the 
order of a few micrometers. Our material system needs to account for the differences in thermal expansion coefficient for sapphire $\left(\sim 8 \times 10^{-6} \mathrm{~K}^{-1}[14]\right)$, GaAs $\left(\sim 6.5 \times 10^{-6} \mathrm{~K}^{-1}[14]\right)$ and GaN $\left(\sim 5.6 \times 10^{-6} \mathrm{~K}^{-1}[15]\right)$. The straight lines (shown in Fig. 3) may belong to such a network of dislocations formed after bonding when the samples were cooled down to room temperature. These dislocations were formed in the GaAs, since they are parallel to either the $[110]_{\mathrm{GaAs}}$ or the $[1-10]_{\text {GaAs }}$ direction.

Finally, we would also have expected to identify a dislocation network that would accommodate the lattice mismatch between GaAs and GaN. When two different materials are fused together lattice mismatch is accommodated by a network of misfit dislocations with edge component in the bonding plane. If the orientations of the two layers are relatively twisted inplane, dislocations with a screw component will also be formed to accommodate the twist. Therefore, a two-dimensional network in the form of a regular grid of misfit dislocations with a Burgers vectors in the bonding plane should appear at the interface when two twisted, lattice mismatched layers are bonded. Such a grid was observed for bonded cubic crystals $[8,13,16]$. However, in our samples no closely-spaced, regular grid of dislocations was observed. This type of dislocation network was also not observed for a bonded sapphire/GaAs interface $[11,12]$. The reasons may have to do with thin amorphous layer at the interface or with relative inertness of the component materials. The high melting point of the GaN or sapphire, relative to the bonding temperature may preclude actual chemical bonding between the GaN (or sapphire) and GaAs. To understand this, further, more detailed studies are necessary.

In summary, the microstructure of the interface formed by direct bonding of GaAs and GaN wafers was investigated using different TEM techniques. The microstructure of the interface of samples fused at $550^{\circ} \mathrm{C}$ and $750^{\circ} \mathrm{C}$ did not differ substantially. Most of the interface 
area was well-bonded, with only a thin (1-2 nm-thick) amorphous layer present at the interface in these well-bonded areas. Cavities, ranging in size from a few tens of nanometers to a few tens of micrometers were also observed at the fused interface. The cavities extended only into the GaAs, and were usually elongated along the $[110]_{\text {GaAs }}$ direction. Plan-view images revealed dislocation networks in the well-bonded areas. One network appeared to arise from unintentional tilts between the fused crystals and the second network was formed due to the mismatch in thermal expansion coefficients between GaAs and GaN. We did not observe dislocation networks originating from lattice mismatch or twist between the fused crystals. These and further studies are critical for the detailed understanding of the mechanisms of wafer bonding, and the application of this process to device fabrication.

This research at LBNL was supported by AFOSR-ISSA-99-0012 under the sponsorship of Dr. G. Witt and at UCSB by the DoD Multidisciplinary University Research Initiative (MURI) program administered by the Office of Naval Research under Grant Nooo14-98-1-0654. The TEM group (J.J. and Z.L.-W.) would like to thank W. Swider for excellent sample preparation, Prof. J. Washburn for fruitful discussions and the National Center for Electron Microscopy at LBNL for the opportunity to use NCEM's facilities. The authors would like to thank A. Huntington and L. Coldren and, A. Stonas and S. DenBaars from UCSB for the growth of GaAs and GaN, respectively. 
References

1. F. A. Kish, D. A. Defevere, D. A. Vanderwater, G. R. Trott, R. J. Weiss, and J. S. Major, Electron. Lett. 30, 1790 (1994).

2. N. M. Margalit, K. A. Black, Y. J. Chiu, E. R. Hegblom, K. Streubel, P. Abraham, M. Anzlowar, J. E. Bowers, and E. L. Hu, Electron. Lett. 34,285 (1998).

3. B. Liu, A. Skakouri, P. Abraham, B.-G. Kim, A. W. Jackson, and J. E. Bowers, Appl. Phys. Lett. 72,2637 (1998).

4. I.-H. Tan, E. L. Hu, and J. E. Bowers, IEEE J. Quantum Electron. 31,1863 (1995).

5. J. Jasinski, Z. Liliental-Weber, S. Estrada, E. Hu, Mater. Res. Soc. Symp. Proc. 722, K7.15 (2002), in print.

6. P.D. Floyd, D.W. Treat, D.P. Hour, IEEE Electron. Lett., 35,2120 (1999).

7. S. Estrada, E. Hu, unpublished.

8. L. Sagalowicz, A. Rudra, E. Kapon, M. Harnmar, F. Salomonsson, A. Black, P.-H. Jouneau, T. Wipijewski, J. Appl. Phys. 87,4135 (2000).

9. P. Kopperschmidt, G. Kastner, D. Hesse, N.D. Zakharov, and U. Gosele, Appl. Phys. Lett. 70, 2972 (1997).

10. N. Y. Jin-Phillipp, W. Sigle, A. Black, D. Babic, J.E. Bowers, E.L. Hu, M. Ruhle, J. Appl. Phys. 89, 1017 (2001).

11. P. Kopperschmidt, G. Kaestner, S. Senz, D. Hesse, U. Goesele, Appl. Phys. A 64, 533 (1997).

12. St. Senz, P. Kopperschmidt, G. Kastner, D. Hesse, J. Mater. Sci. 33, 2073 (1998).

13. G. Patriarche, F. Jeannes, J.-L. Oudar, F. Glas, J. Appl. Phys. 82,4892 (1997). 
14. Y.S. Touloukian, R.K. Kirby, R.E. Taylor, T.Y.R. Lee, 'Thermal Expansion-Nonmetallic Solids', Plenum, NY 1977

15. H. Kinoshita, S. Otani, S. Kamiyama, H. Amano, I. Akasaki, J. Suda, H. Matsunami, Jpn. Journ. Appl. Phys. 40, L1280 (2001)

16. M. Benamara, A. Rocher, A. Laporte, G. Sarrabayrousse, L. Lescouzeres, A. PeyreLavigne, M. Fnaiech, and A. Claverie, Mater. Res. Soc. Symp. Proc. 378, 863 (1995). 


\section{FIGURE CAPTIONS}

Fig.1. Schematic drawing of the: (a) starting materials, (b) structure after fusion and GaAs substrate removal.

Fig. 2. HREM images recorded in cross-section at the wafer-fused GaAs/GaN interface in a (a) well-bonded region, and (b)-(c) areas containing cavities. (d) Plan-view TEM image of an area containing cavities.

Fig. 3. (a) and (b) bright field TEM images of a plan-view specimen, at different magnifications. Pairs of arrow shown in (a) indicate three dislocation lines probably formed when the sample was cooled down to room temperature. 


\begin{tabular}{|c|c|}
\multicolumn{1}{c|}{ GaAs by MBE } & GaN by MOCVD \\
\hline $1 \mu \mathrm{m}$ pGaAs & $>2 \mu \mathrm{m} \mathrm{nGaN}$ \\
\hline $0.3 \mu \mathrm{m}$ AlAs etch stop \\
\hline $\begin{array}{c}(100) \text { nGaAs } \\
\text { substrate }\end{array}$ & $\begin{array}{c}(0001) \text { sapphire } \\
\text { substrate }\end{array}$ \\
\hline
\end{tabular}

(a)

\begin{tabular}{|c|}
\hline $1 \mu \mathrm{m}$ pGaAs \\
\hline$>2 \mu \mathrm{m} \mathrm{nGaN}$ \\
\hline $\begin{array}{c}\text { (0001) sapphire } \\
\text { substrate }\end{array}$ \\
\hline
\end{tabular}

(b)

J. Jasinski et al., Fig. 1 

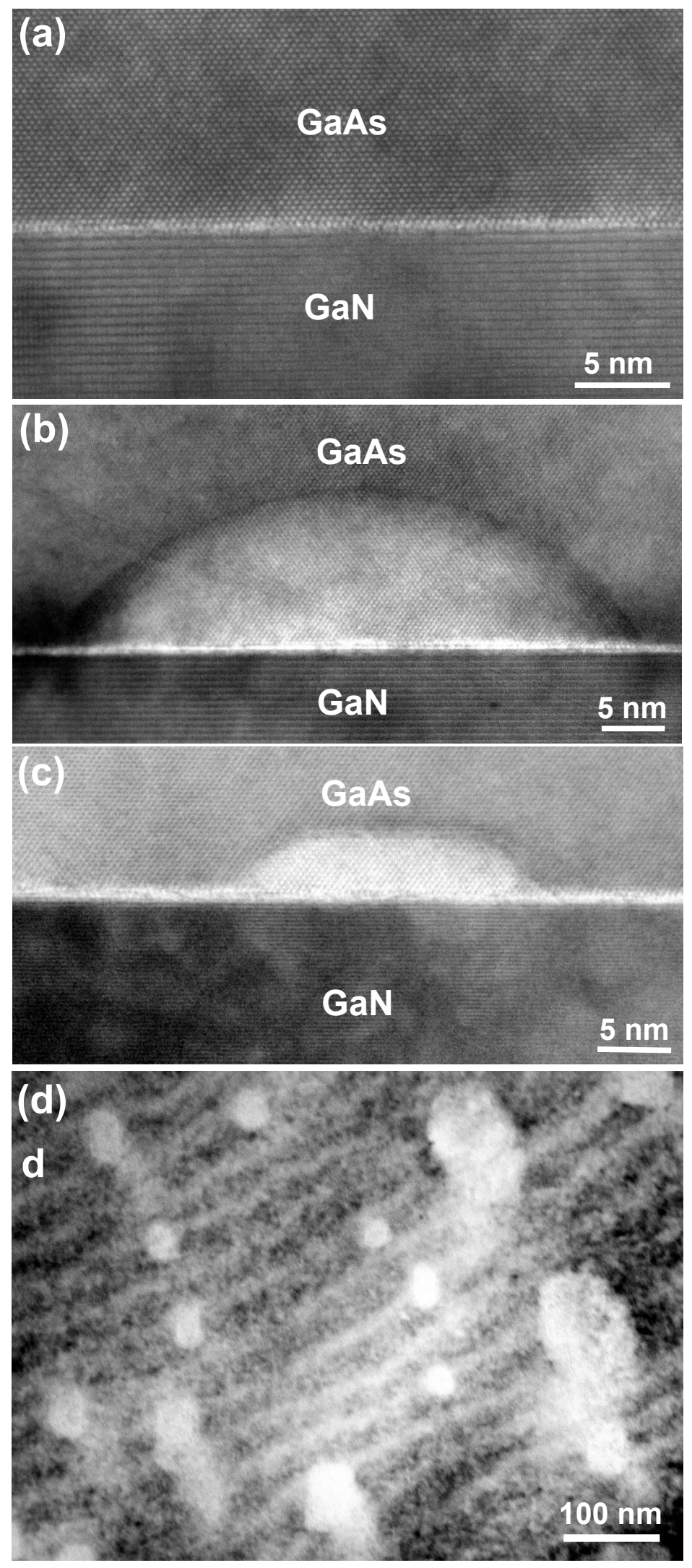

J. Jasinski et al., Fig. 2 

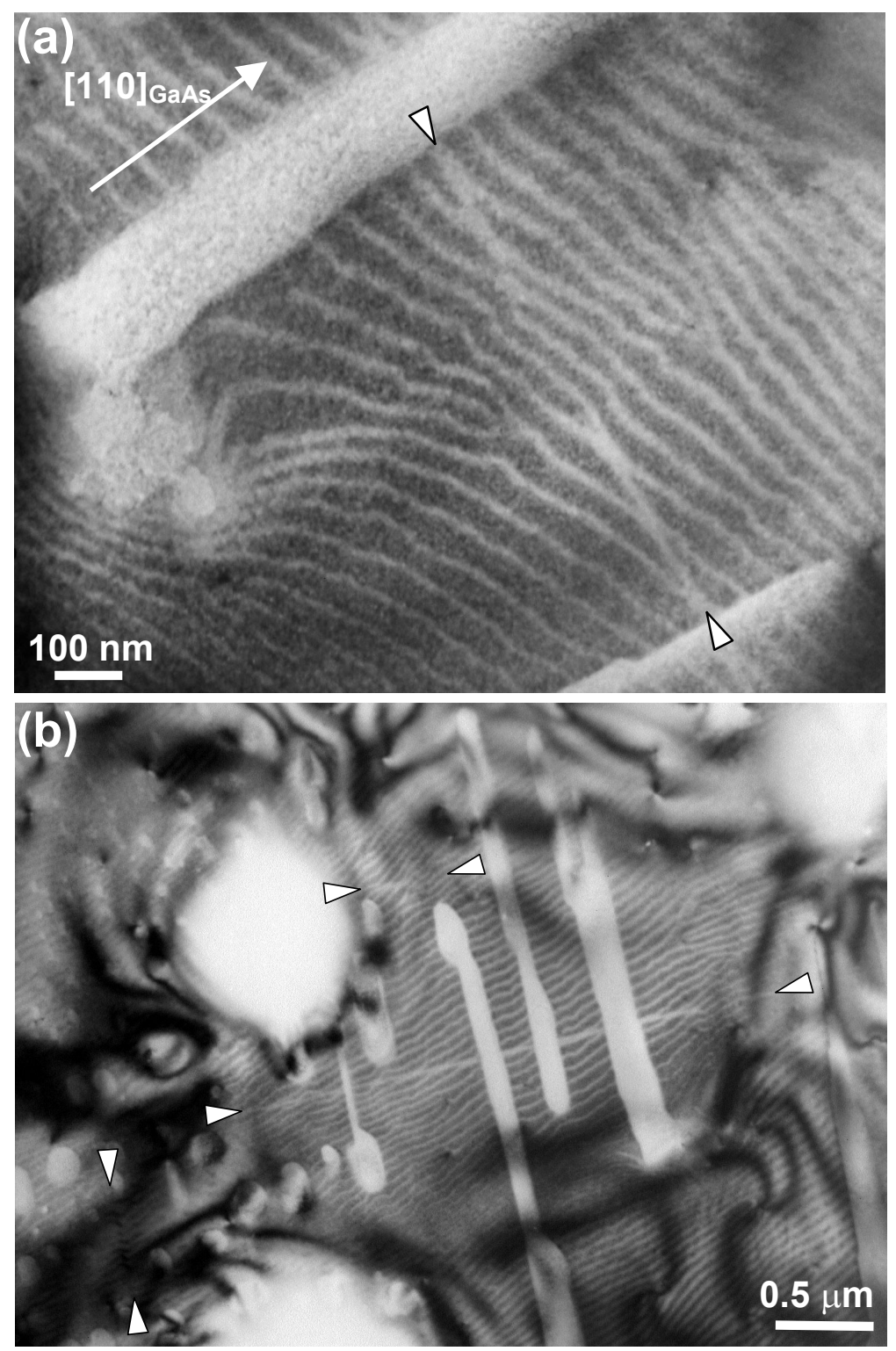

J. Jasinski et al., Fig. 3 